\title{
Sparse Channel Estimation based on Compressed Sensing for Ultra WideBand Systems
}

\author{
Eva Lagunas* and Montse Nájar *† \\ *Universitat Politecnica de Catalunya (UPC), Barcelona, Spain \\ ${ }^{\dagger}$ Centre Tecnologic de Comunicacions de Catalunya (CTTC), Castelldefels, Spain \\ Email: eva.lagunas@upc.edu,montse.najar@upc.edu
}

\begin{abstract}
Channel estimation for purposes of equalization is a long standing problem in signal processing. Wireless propagation is characterized by sparse channels, that is channels whose time domain impulse response consists of few dominant multipath fingers. This paper examines the use of Compressed Sensing (CS) in the estimation of highly sparse channels. In particular, a new channel sparse model for ultra-wideband (UWB) communication systems based on the frequency domain signal model is presented. A new greedy algorithm named extended OMP (eOMP) is proposed to reduce the false path detection achieved with classical Orthogonal Matching Pursuit (OMP) allowing better time of arrival (TOA) estimation.
\end{abstract}

Index Terms-Channel estimation, Ultra WideBand technology, Compressed Sensing.

\section{INTRODUCTION}

Reflection, diffraction and scattering from surrounding objects are typical effects suffered by signals while propagate through a wireless channel. Because of these effects, the transmitted signal arrives at the receiver as a superposition of multiple attenuated and delayed copies of the original signal. However, multipath can be seen both as a curse or as a blessing from a communications point of view depending on the amount of Channel State Information (CSI) available to the system. If the channel characteristics are known at the receiver, it can be effectively use to improve the communications performance.

On the other hand, UWB communications [1] has emerged as a promising technology for wireless communications. Designed for low-power, short-range, wireless personal area networks, UWB is the leading technology for freeing people from wires, enabling wireless connection of multiple devices for transmission of high-bandwidth data.

The transmission of ultrashort pulses in UWB leads to several desirable characteristics such as the rich multipath diversity introduced by the large number of propagation paths existing in a UWB channel. The rich multipath coupled with the fine time resolution of UWB create a challenging channel estimation problem. Fortunately, wireless channel can often be modeled as a sparse channel in which the delay spread

E. Lagunas was supported by the Catalan Government under grant FI-DGR 2010.

This work was partially supported by the Catalan Government under grant 2009 SGR 891, by the Spanish Government under project TEC200806327- C03 (MULTI-ADAPTIVE), by the European Cooperation in Science and Technology under project COST Action IC0902 and by the European Commission under project NEWCOM++ (ICT-FP7-216715). could be very large compared with the number of significant paths. Our goal herein is to exploit this sparse structure of the wireless channel impulse reponse in order to improve the channel estimation by means of the emerging CS theory.

CS [2] is a novel sampling paradigm that goes further than Shannon's theorem. The idea is to perfectly recover the signal using far fewer samples of measurements than traditional methods. CS allows to compress the data while is sampled. It originates from the idea that it is not necessary to invest a lot of power into observing the entries of a sparse signal because most of them will be zero.

It is proved that conventional channel estimation methods provide higher errors because they ignore the prior knowledge of the sparseness [3]. The sparse channel estimation problem is faced in [4] under a time domain sparse model point of view. In [4] a suitable dictionary formed by delayed versions of the UWB transmitted pulse is defined in order to better match the UWB signal. However, the spike basis achieves maximal incoherence with the Fourier basis [5] and is for that reason that seems more convenient to work with frequency domain measurements. In [6] the sparse model is defined in the frequency domain. To ensure that every measurement counts, they propose to pre-modulate the input signal with a spread spectrum sequence before the Fourier transformation.

Here, a sparse channel estimation approach is developed based on the sparse frequency domain model of the UWB signals without pre-modulation. We propose exploiting the sparse nature of the channel through the use of a new greedy algorithm named extended OMP (eOMP) in order to reduce the false path detection probability achieved with classical OMP and to derive an improved TOA estimation. TOA stands out as the most suitable signal parameter to be used for positioning with UWB devices and is currently the focus of intense research in the UWB research community. The eOMP algorithm will be tested with other models in the literature [4] [6] to show that its performance is not limited to the model proposed here.

In this paper we first describe the classic UWB signal model in Section II. In Section III the sparse frequency domain model and the extended OMP (eOMP) are introduced. Simulation results and Conclusions are given in Section IV and Section $\mathrm{V}$, respectively. 


\section{UWB Signal Model}

The transmitted UWB signal model can be written as,

$$
s(t)=\sum_{k=0}^{\infty} \sum_{j=0}^{N_{f}-1} a_{k} p\left(t-j T_{f}-k T_{\text {sym }}\right)
$$

where the data $a_{k} \in \pm 1$ is the k-th transmitted bit, $T_{\text {sym }}$ is the symbol duration and $T_{f}=T_{\text {sym }} / N_{f}$ is the pulse repetition period. To simplify notation, in the following it is assumed $a_{k}=1$.

Signal $s(t)$ propagates through an L-path fading channel whose response to $p(t)$ is $\sum_{l=0}^{L-1} h_{l} p\left(t-\tau_{l}\right)$. The received waveform can be written as

$$
r(t)=\sum_{k=0}^{\infty} \sum_{j=0}^{N_{f}-1} \sum_{l=0}^{L-1} h_{l} p\left(t-T_{k}^{j}-\tau_{l}\right)+w(t)
$$

where $w(t)$ is thermal noise with two-sided power spectral density $N o / 2$ and $T_{k}^{j}=j T_{f}+k T_{\text {sym }}$. The signal associated to the $\mathrm{j}$-th transmitted pulse corresponding to the $\mathrm{k}$-th symbol, in the frequency domain yields

$$
Y_{j}^{k}(w)=\sum_{l=0}^{L-1} h_{l} S_{j}^{k}(w) e^{-j w \tau_{l}}+V_{j}^{k}(w)
$$

with

$$
S_{j}^{k}(w)=P(w) e^{-j w\left(k N_{f}+j\right) T_{f}}
$$

where $P(w)$ denotes the Fourier Transform of the pulse $p(t)$ and $V_{j}^{k}(w)$ is the noise in the frequency domain associated to the $\mathrm{j}$-th frame interval correspondig to the $\mathrm{k}$-th symbol. Sampling (3) at $w_{m}=w_{0} m$ for $m=0,1, \ldots, M-1$ where $w_{0}=\frac{2 \pi}{T_{f}}$ and rearranging the frequency domain samples $Y_{j}^{k}[m]$ into the vector $\mathbf{Y}_{j}^{k} \in \mathbb{C}^{M \times 1}$ yields

$$
\mathbf{Y}_{j}^{k}=\sum_{l=0}^{L-1} h_{l} \mathbf{S}_{j}^{k} \mathbf{e}_{\tau_{l}}+\mathbf{V}_{j}^{k}=\mathbf{S}_{j}^{k} \mathbf{E h}+\mathbf{V}_{j}^{k}
$$

where the matrix $\mathbf{S}_{j}^{k} \in \mathbb{C}^{M \times M}$ is a diagonal matrix whose components are the frequency samples of $S_{j}^{k}(w)$ and the matrix $\mathbf{E} \in \mathbb{C}^{M \times L}$ contains the delay-signature vectors associated to each arriving delayed signal

$$
\mathbf{E}=\left[\begin{array}{lllll}
\mathbf{e}_{\tau_{0}} & \ldots & \mathbf{e}_{\tau_{l}} & \ldots & \mathbf{e}_{\tau_{L-1}}
\end{array}\right]
$$

with $\mathbf{e}_{\tau_{l}}=\left[\begin{array}{llll}1 & e^{-j w_{0} \tau_{l}} & \ldots & e^{-j w_{0}(M-1) \tau_{l}}\end{array}\right]^{T}$. The channel fading coefficients are arranged in the vector $\mathbf{h}=$ $\left[\begin{array}{lll}h_{0} & \ldots & h_{L-1}\end{array}\right]^{T} \in \mathbb{R}^{L \times 1}$, and the noise samples in vector $\mathbf{V}_{j}^{k} \in \mathbb{C}^{M \times 1}$.

\section{EXTENDED OMP IN FREQUenCy Domain}

A proper sparse representation of the channel is required in order to easily apply the CS theory. A tutorial overview of some of the basic developments in CS can be found in [5]. The expression in (5) free of noise can be extended and reformulated as,

$$
\mathbf{Y}_{j}^{k}=\mathbf{B}_{j}^{k} \mathbf{h}_{e}=\mathbf{S}_{j}^{k} \mathbf{E}_{e} \mathbf{h}_{e}
$$

The main difference between (7) and (5) is the extended matrix $\mathbf{E}_{e}$, which is an $M \times M$ extended delay-matrix which contains not only the $\mathrm{L}$ delay-signature vectors corresponding to the multipath, but also $M-L$ delay-signature vectors with no channel contribution (see Fig. 1). Therefore, vector $\mathbf{h}_{e}$ is an $L$ sparse vector whose elements different from zero correspond to the original channel coefficients, that is, calling $\mathbf{e}_{\tau_{m}}$ the m-th column of $\mathbf{E}_{e}$, when

$$
\mathbf{e}_{\tau_{m}}=\mathbf{e}_{\tau_{l}} \quad \text { for } \quad l=0, \ldots, L-1
$$

Note that the dimension $M$ will determine the path resolution.

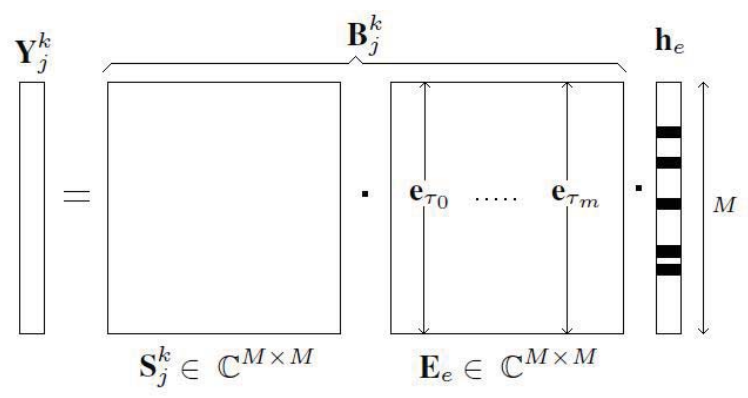

Fig. 1. Sparse Sructure of the channel

In a typical CS notation, $\mathbf{h}_{e}$ can be identified as the $L$-sparse vector and $\mathbf{B}_{j}^{k}$ as the dictionary where the channel becomes sparse. In order to compress the frequency domain samples a widely used random matrix $\mathbf{C}_{f} \in \mathbb{R}^{N \times M}$ with entries i.i.d. taken from a normal distribution with zero-mean and unit variance is used.

$$
\mathbf{Y}_{c}=\mathbf{C}_{f} \mathbf{Y}_{j}^{k}=\mathbf{C}_{f} \mathbf{B}_{j}^{k} \mathbf{h}_{e}
$$

where $\mathbf{Y}_{c}$ is the $N \times 1$ vector of measurements. $\mathbf{C}_{f}$ is known as measurement matrix and it has rank $N$ lesser than the rank of the signal which is equal to $M$. Thus, the $N \times M$ matrix $\mathbf{C}_{f}$ is projecting the signal $\mathbf{Y}_{j}^{k}$. Randomness in the measurement matrix can lead to very efficient sensing mechanisms. It has been shown that random matrices are largely incoherent with any fixed basis (which is one of the principles of CS).

Therefore, the sparse channel estimation $\hat{\mathbf{h}}_{e}$ can be obtained from the compressed samples $\mathbf{Y}_{c}$ applying sparse signal reconstruction techniques. The sparse signal recovery problem is formulated as,

$$
\min _{\hat{\mathbf{h}}_{e} \in \mathbb{R}^{M}}\left\|\hat{\mathbf{h}}_{e}\right\|_{l_{1}} \quad \text { s.t. } \quad \mathbf{Y}_{c}=\mathbf{C}_{f} \mathbf{B}_{j}^{k} \hat{\mathbf{h}}_{e}
$$

where $\left\|\hat{\mathbf{h}}_{e}\right\|_{l_{1}}=\sum_{i=1}^{M}\left|\hat{h}_{e}(i)\right|$. Note that the only prior knowledge required is that $\mathbf{h}_{e}$ is sparse. Reconstruction then only requires the space in which the signal is sparse.

There are many approaches discussed in literature for solving (10). Currently, the two most popular approaches are matching pursuit (MP) [7] and basis pursuit (BP) [8]. With MP (and one of its variants OMP) the sparse signal is iteratively built up by selecting the atom that maximally improves the representation at each iteration. On the other hand, BP directly 
looks for the vector that minimize the $l_{1}$-norm coefficients, which is computationally expensive. Here, OMP [9] is used to achieve faster and more efficient reconstruction. However, imperfections between the assumed model and the received signal can cause false path detection, leading to a wrong TOA estimation. To improve the TOA estimation but preserving the performance of channel estimator it is proposed an extended OMP (eOMP) (Algorithm 1). Both OMP and eOMP are iterative greedy algorithms that select at each step the dictionary element best correlated with the residual part of the signal. Then they produce a new approximation by projecting the signal onto the dictionary elements that have already been selected. The main difference between OMP and eOMP is that eOMP not only pick the column of the dictionary that is most strongly correlated but also the $2 k+1$ neighbors of it. To obtain the final values of the non-zero elements of the sparse vector the same step with only the most strongly correlated element is computed in parallel. The running time of the OMP and eOMP is dominated by Step 2, whose total cost is $O(m N M)$ where $m$ is the number of iterations.

\section{Simulation Results}

For numerical evaluation of the algorithm we consider the channel models developed within the framework of the IEEE 802.15.4a. In particular it is used the CM1 Residential LOS channel model. All simulations are given for 100 independent channel realizations. $\mathrm{M}$ is fixed at 768 and the compression rate is expressed with $\rho=\frac{N}{M}$ (meaning $\rho<<1$ high undersampling). The pulse duration is equal to $0.77 \mathrm{~ns}$ (which theoreticaly correspond to a Nyquist compression when $\rho=$ 0.33 ). The number of multipath components $L$ that form the UWB channel can be quite large, however many of those paths are negligible. Therefore, we limit ourselves to estimate the $L_{c}$ most significant paths which are the ones capturing $80 \%$ of the channel energy.

The quality of the channel estimation is evaluated with the RMSE computed as $\sqrt{\frac{1}{M} \mathbf{e}^{H} \mathbf{e}}$, where the error $\mathbf{e}$ is defined as $\mathbf{e}=\operatorname{ifft}\left(\mathbf{B}_{j}^{k} \hat{\mathbf{h}}_{e}\right)-\mathbf{r}_{w n}$, with $\mathbf{r}_{w n}$ being the received signal without noise.

\section{A. eOMP vs classical OMP}

A comparison with the classical OMP will be developed in order to show the performance of the proposed eOMP. Fig.2 depicts the RMSE of the reconstructed signal using OMP and eOMP. Examining the reconstruction error of each algorithm as a function of the SNR, we observe that OMP exhibits slightly better performance than eOMP. However, the biggest diference is of the order of $10^{-4}$ and it decreses as the noise level increases. Although OMP obtains lots of false path detections (see this in Fig.3), they have low contribution to the overall channel. Thus, the contribution of the false paths detected by OMP affects less on the reconstruction than missing paths with big contribution. That is what is happening when using eOMP whatever the value of $\mathrm{k}$ : we have no false path detection but probably we are missing some paths with significant contribution to the overall channel. It is also

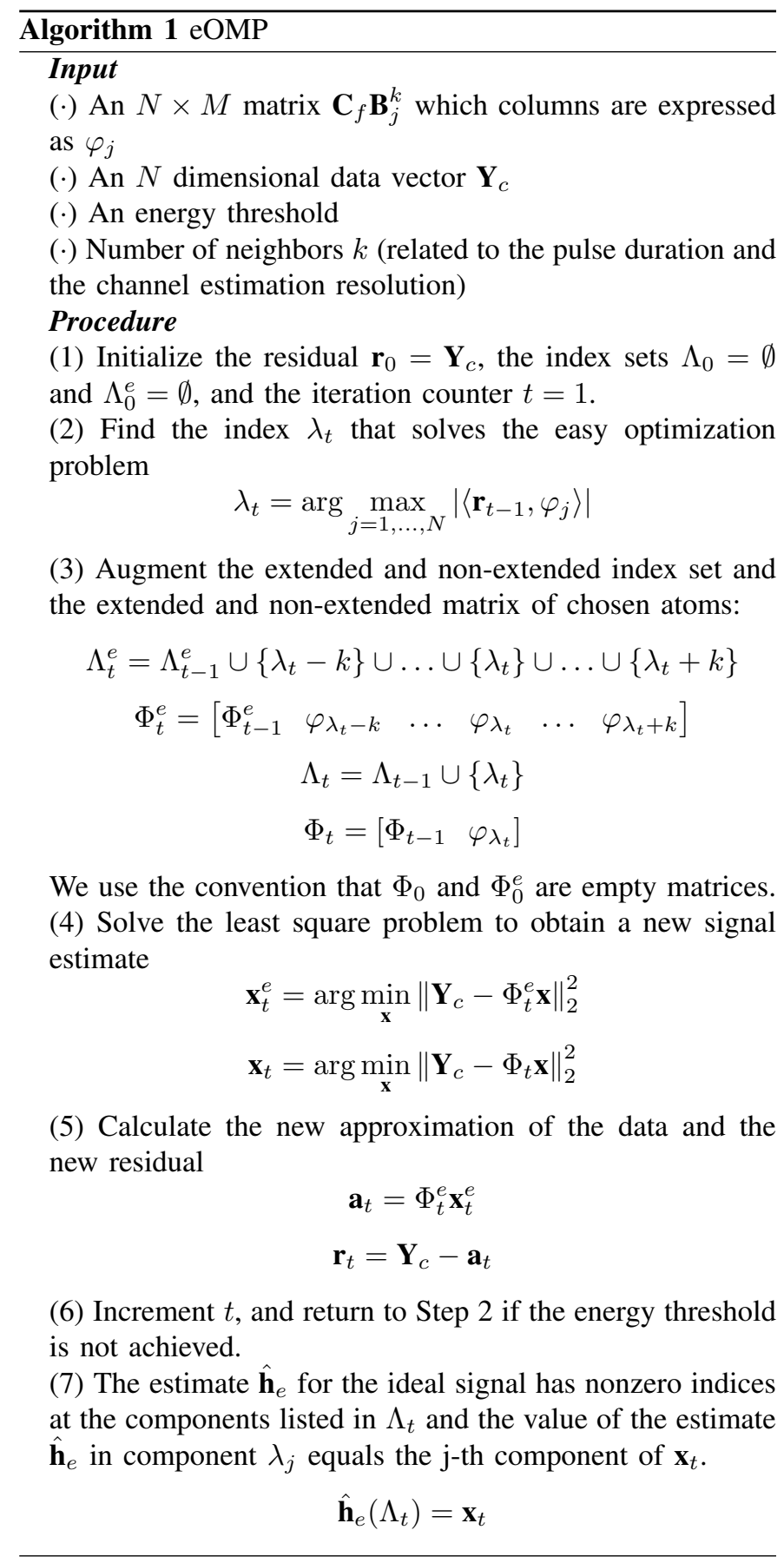

confirmed by the fact that the error increases as the value of $k$ increases, meaning that the higher the value of $\mathrm{k}$, the more the missing paths.

To confirm that the proposed eOMP reduces the false path detection let us now study the probability of false path detection of eOMP compared with the classical OMP. Fig. 3 depicts the false path detection probability using OMP and eOMP. The results show that the false path detection probability is significantly reduced when using eOMP whatever the value of $k$.

One thing that attracts our attention is the peak of false 


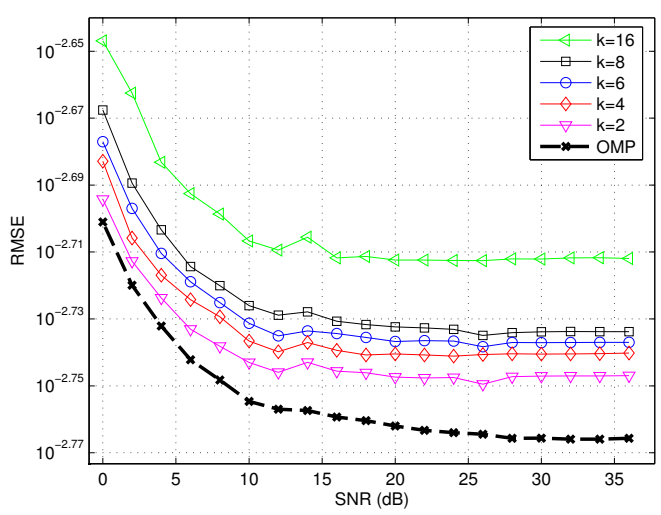

Fig. 2. RMSE of the sparse channel estimation with $\rho=0.333$

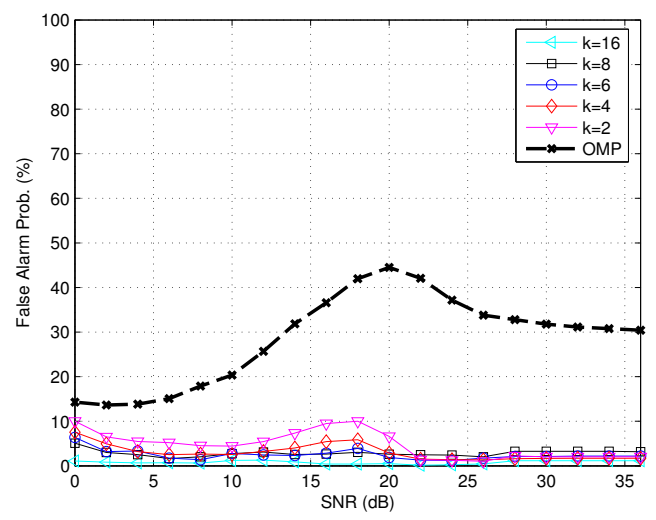

Fig. 3. False path detection probability with $\rho=0.333$

alarms around the $20 \mathrm{~dB}$ of SNR. The peak can be perfectly seen in the case of OMP and, although not distinguishable very well, it persists in the case eOMP. To understand what is happening, Fig. 4 shows the number of iterations required for OMP and eOMP. The first conclusion extracted from Fig.4 is that another advantage of eOMP compared with classical OMP is its high speed. In eOMP fewer steps are required to converge. This is a direct consequence of the eOMP design itself. The stopping criteria is a function of the number of neighbors used since it is based on the recovered signal energy, which is computed using all the neighbors. Returning to the peak of false alarms observed in Fig. 4, we can see that the iterations also have a peak at the same point of $S N R=20 \mathrm{~dB}$. This demonstrates that it is possible that we are running the algorithm too long, when, in fact, we should stop the algorithm much earlier. The design of a convenient method for deciding when to halt the iteration is a diffcult task. A natural stopping rule for the subset selection problem is not immediately apparent. Here an intuitive stopping criteria based on the energy of the output estimate signal is being used but, apparently it is a point for further research.

To show that the classical OMP provides better correct path detection let us now study the probability of detecting a true path of OMP compared with the proposed eOMP. Fig.5 provides the simulation results regarding the correct

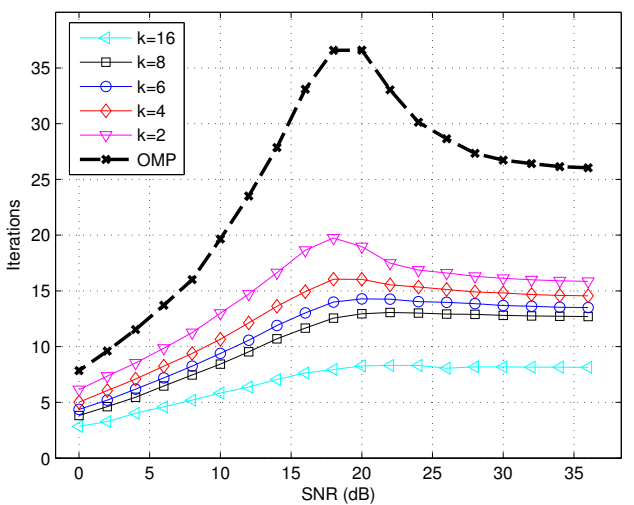

Fig. 4. Number of iterations with $\rho=0.333$

path detection probability using OMP and eOMP. Prior to the simulations we have commented that a high value on the parameter $k$ could cause miss detection of true paths. This is the reason why in Fig.5 we have obtained higher correct path detection when $k$ is low. The fact that OMP is detecting more true paths than eOMP is also proved with these results. Thus, OMP always exhibits more correct path detection than eOMP because the more paths detected, the more likely hitting a true one.

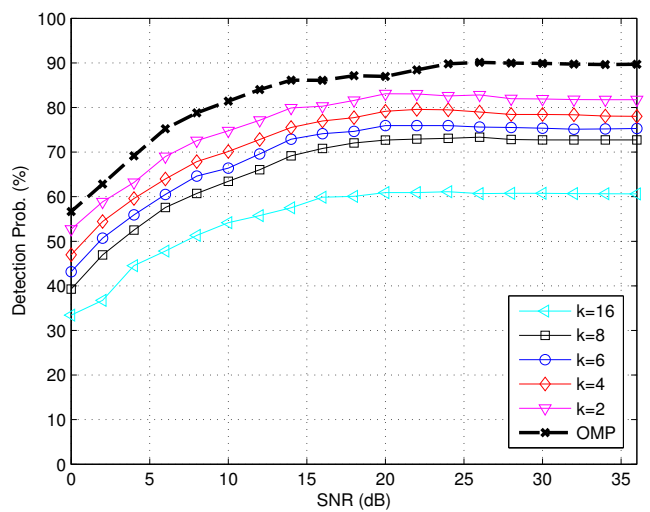

Fig. 5. Correct path detection probability with $\rho=0.333$

Finally, let us study the performance of our main objective: the TOA estimation. The RMSE of the estimated TOA using OMP and eOMP $(\mathrm{k}=2)$ are depicted in Fig. 6. The simulation result shows that with eOMP $(\mathrm{k}=2)$ an accuracy of few centimeters is achieved while the OMP accuracy is about one meter, and this is a direct consequence of cleanning the first estimated path.

From now on, eOMP will be used in future simulations.

\section{B. eOMP with other models present in the literature}

The perfomance of the eOMP is tested with the time domain sparse model proposed in [4] where the dictionary used is a circulant matrix $\mathbf{P} \in \mathbb{R}^{M \times M}$ whose columns are shifted replicas of the mother pulse $p(t)$. The compressed samples 


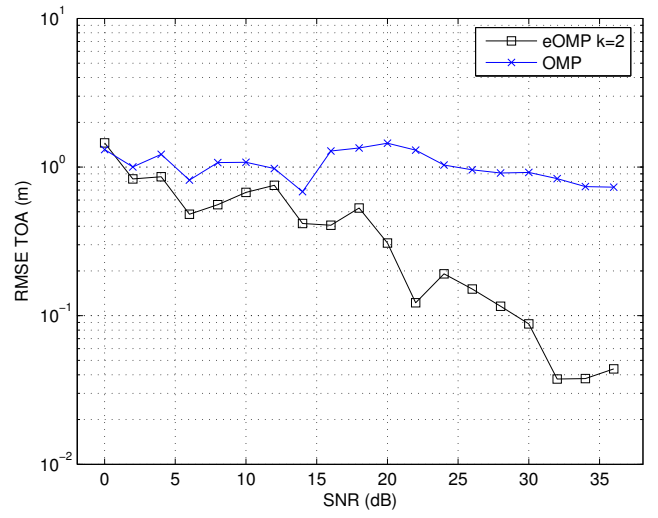

Fig. 6. TOA estimation for $\rho=0.167$

are obtained with a random measurement matrix as in (9),

$$
\mathbf{y}_{c}=\mathbf{C}_{t} \mathbf{P} \hat{\mathbf{h}}_{e}
$$

where $\mathbf{C}_{t} \in \mathbb{R}^{N \times M}$. In this case the RMSE is obtained with the following error definition,

$$
\mathbf{e}=\mathbf{P} \hat{\mathbf{h}}_{e}-\mathbf{r}_{w n}
$$

Fig. 7 depicts the RMSE of the recovered signal using the channel estimation presented here compared with [4]. At the Nyquist compression rate, the RMSE remains the same for both approaches. As the compression rate decrease, the errors in the time domain seem to increases slightly faster than in the frequency domain for high SNR. However, the differences are negligible and the conclusion is that eOMP works well regardless of the sparse signal model.

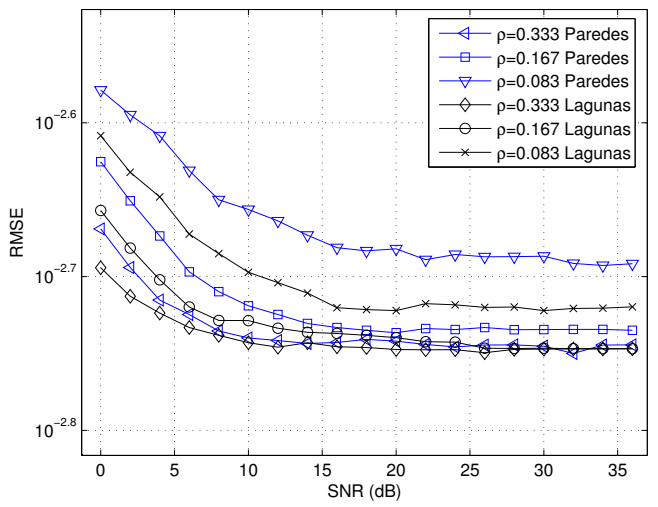

Fig. 7. RMSE of the recovered signal compared with the time domain approach in [4]

Another frequency domain model presented in [6] has been studied. In [6], the spectrum is spread pre-modulating the input signal with a pseudorandom sequence $p[n]$ before the Fourier transformation ensuring that every measurement carries information,

$$
\mathbf{y}_{c}=\mathbf{R F} \operatorname{diag}(p[n]) \mathbf{P h}_{e}
$$

where $\mathbf{F}$ is the column normalized DFT matrix and $\mathbf{R}$ is the sub-sampling operator. Results depicted in Fig. 8 show that the estimation error with the new model slightly outperforms [6] whatever the compression rate is. However, as happened with [4], the differences are difficult to see and, again, we can state that the eOMP performance does not depend on the sparse signal model considered.

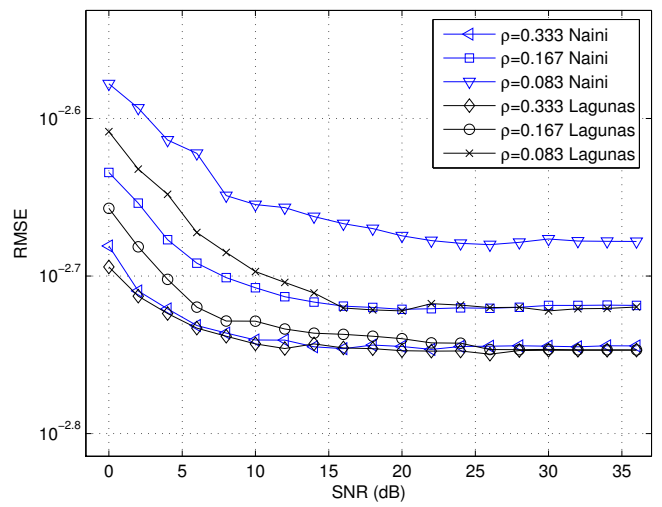

Fig. 8. RMSE of the recovered signal compared with the approach in [6]

\section{CONCLUSIONS}

A frequency domain UWB sparse channel estimator based on CS theory has been presented and a new algorithm named eOMP is proposed. Simulation results proved that eOMP improves the classical OMP TOA estimation by reducing the false path detection probability. On the other hand, simulation results carried out with eOMP but with different sparse signal models [4] [6] have shown that the new eOMP performance does not depend on the sparse signal model considered.

\section{REFERENCES}

[1] X. Shen, M. Guizani, H. Chen, R. C. Qui and A. Molisch, Ultra-Wideband Wireless Communications, IEEE J. Select. Areas on Communications, Vol. 24, 2006.

[2] D. L. Donoho, Compressed Sensing, IEEE Trans. Information Theory, Vol. 52, no. 4, pp. 1289-1306, Apr 2006.

[3] F. Wan, W. P. Zhu and M. N.S. Swamy, Semi-Blind Most Significant Tap Detection for Sparse Channel Estimation of OFDM Systems, IEEE Trans. Circuits and Systems, Vol. 57, no. 3, pp. 703-713, Mar 2010.

[4] J. L. Paredes, G. R. Arce and Z. Wang, Ultra-Wideband Compressed Sensing: Channel Estimation, IEEE J. Select. Top. Sig. Proc., Vol. 1, no. 3, pp. 383-395, Oct 2007.

[5] E. J. Candes and M. B. Wakin, An Introduction to Compressed Sampling, IEEE Sig. Proc. Magazine, Vol. 25, no. 2, pp. 21-30, Mar 2008.

[6] F. M. Naini, R. Gribonval, J. Jacques and P. Vandergheynst, Ultra-Wide Bandwidth Time-Hopping Spread-Spectrum Impulse Radio for Wireless Multiple-Acces Communications, Proc. ICASSP, pp. 2877-2880, 2009.

[7] S. G. Mallat and Z. Zhang, Matching Pursuits with Time-Frequency Dictionaries, IEEE Trans. Sig. Proc., Vol. 41, no. 12, pp. 3397-3415, Dec 1993.

[8] S. S. Chen, D. L. Donoho and M. A. Saunders, Atomic Decomposition by Basis Pursuit, Soc. for Ind. and App. Math. Review, Vol. 43, no. 1, pp. 129-159, 2001.

[9] J. A. Tropp and A. C. Gilbert, Signal Recovery From Random Measurements Via Orthogonal Matching Pursuit, IEEE Trans. Information Theory, Vol. 53, no. 12, pp. 4655-4666, Dec 2007. 